\title{
ORIENTED INCIDENCE COLOURINGS OF DIGRAPHS
}

\author{
Christopher Duffy \\ Department of Mathematics and Statistics \\ University of Saskatchewan \\ Saskatoon, Canada \\ e-mail: christopher.duffy@usask.ca \\ Gary MaCGiLlivray \\ Department of Mathematics and Statistics \\ University of Victoria \\ Victoria, Canada \\ e-mail: gmacgill@math.uvic.ca \\ Pascal OChem \\ LIRMM, CNRS, \\ Université de Montpellier, \\ Montpellier, France \\ e-mail: pascal.ochem@lirmm.fr
}

AND

AndRÉ RAspaud

LaBRI UMR CNRS 5800

Université Bordeaux 1,

Bordeaux, France

e-mail: raspaud@labri.fr

\begin{abstract}
Brualdi and Quinn Massey [6] defined incidence colouring while studying the strong edge chromatic index of bipartite graphs. Here we introduce a similar concept for digraphs and define the oriented incidence chromatic number. Using digraph homomorphisms, we show that the oriented incidence chromatic number of a digraph is closely related to the chromatic number of the underlying simple graph. This motivates our study of the oriented incidence chromatic number of symmetric complete digraphs. We give
\end{abstract}


upper and lower bounds for the oriented incidence chromatic number of these graphs, as well as digraphs arising from common graph constructions and decompositions. Additionally we construct, for all $k \geqslant 2$, a target digraph $H_{k}$ for which oriented incidence $k$ colouring is equivalent to homomorphism to $H_{k}$.

Keywords: digraph homomorpism, graph colouring, incidence colouring, computational complexity.

2010 Mathematics Subject Classification: 05C15.

\section{REFERENCES}

[1] K. Appel, W. Haken and J. Koch, Every planar map is four colorable. Part rm II: Reducibility, Illinois J. Math. 21 (1977) 491-567.

[2] J. Bang-Jensen, P. Hell and G. MacGillivray, The complexity of colouring by semicomplete digraphs, SIAM J. Discrete Math. 1 (1988) 281-298. doi:10.1137/0401029

[3] L. Barto, M. Kozik and T. Niven, The CSP dichotomy holds for digraphs with no sources and no sinks (a positive answer to a conjecture of Bang-Jensen and Hell), SIAM J. Comput. 38 (2009) 1782-1802. doi:10.1137/070708093

[4] J. Bensmail, C. Duffy and S. Sen, Analogues of cliques for $(m, n)$-colored mixed graphs, Graphs Combin. 33 (2017) 735 - 750. doi:10.1007/s00373-017-1807-2

[5] J.A. Bondy and U.S.R. Murty, Graph Theory (Springer-Verlag, London, 2008).

[6] R.A. Brualdi and J.J. Quinn Massey, Incidence and strong edge colorings of graphs, Discrete Math. 122 (1993) 51-58.

doi:10.1016/0012-365X(93)90286-3

[7] B. Courcelle, The monadic second order logic of graphs VI: on several representations of graphs by relational structures., Discrete Appl. Math. 54 (1994) 117-129. doi:10.1016/0166-218X(94)90019-1

[8] W.D. Fellner, On minimal graphs, Theoret. Comput. Sci. 17 (1982) 103-110. doi:10.1016/0304-3975(82)90135-9

[9] R.L. Graham and N.J.A. Sloane, Lower bounds for constant weight codes, IEEE Trans. Inform. Theory 26 (1980) 37-43.

doi:10.1109/TIT.1980.1056141

[10] P. Hell and J. Nešetřil, On the complexity of H-coloring, J. Combin. Theory Ser. B 48 (1990) 92-110. doi:10.1016/0095-8956(90)90132-J

[11] M. Hosseini Dolama, E. Sopena and X. Zhu, Incidence coloring of $k$-degenerated graphs, Discrete Math. 283 (2004) 121-128. doi:10.1016/j.disc.2004.01.015 
[12] W. Klostermeyer and G. MacGillivray, Homomorphisms and oriented colorings of equivalence classes of oriented graphs, Discrete Math. 274 (2004) 161-172. doi:10.1016/S0012-365X(03)00086-4

[13] A.V. Kostochka, E. Sopena and X. Zhu, Acyclic and oriented chromatic numbers of graphs, J. Graph Theory 24 (1997) 331-340. doi:10.1002/(SICI)1097-0118(199704)24:4〈331::AID-JGT5〉3.0.CO;2-P

[14] G. MacGillivray and K. Sherk, A theory of 2-dipath colourings, Australas. J. Combin. 60 (2014) 11-26.

[15] G. MacGillivray and J.S. Swarts, Obstructions to homomorphisms involving the graft extension, manuscript.

[16] T.H. Marshall, Homomorphism bounds for oriented planar graphs, J. Graph Theory 55 (2007) 175-190. doi:10.1002/jgt.20233

[17] J. Nešetřil and A. Pultr, On classes of relations and graphs determined by subobjects and factorobjects, Discrete Math. 22 (1978) 287-300.

doi:10.1016/0012-365X(78)90062-6

[18] S. Sen, Maximum order of a planar oclique is 15, in: Proc. 23rd International Workshop on Combinatorial Algorithms, W.F. Smyth and S. Arumugam (Ed(s)), (Springer, 2012) 130-142. doi:10.1007/978-3-642-35926-2_16

[19] E. Sopena, Oriented graph coloring, Discrete Math. 229 (2001) 359-369. doi:10.1016/S0012-365X(00)00216-8

[20] P.K. Sun, Incidence coloring of regular graphs and complement graphs, Taiwanese J. Math. 16 (2012) 2289-2295. doi:10.11650/twjm/1500406852

[21] E. Welzl, Color-families are dense, Theoret. Comput. Sci. 17 (1982) 29-41. doi:10.1016/0304-3975(82)90129-3

[22] J. Wu, Some results on the incidence coloring number of a graph, Discrete Math. 309 (2009) 3866-3870. doi:10.1016/j.disc.2008.10.027

[23] K. Young, 2-Dipath and Proper 2-Dipath Colouring (Masters Thesis, University of Victoria, 2009).

Received 12 September 2016

Revised 12 July 2017

Accepted 13 July 2017

\section{Appendix}




\begin{tabular}{c|l} 
Colour Class & Vertex List \\
\hline 1 & $(1,(7,1)),(3,(3,2)),(2,(4,2)),(3,(4,3)),(3,(3,5))$, \\
& $(5,(4,5)),(5,(6,5)),(3,(3,7)),(2,(7,2)),(5,(7,5))$, \\
& $(6,(7,6)),(1,(6,1)),(6,(6,4)),(6,(6,2))$ \\
\hline 2 & $(6,(1,6)),(7,(1,7)),(2,(3,2)),(4,(3,4)),(4,(4,1))$, \\
& $(4,(4,2)),(2,(5,2)),(4,(4,5)),(6,(5,6)),(4,(4,7))$, \\
& $(7,(3,7)),(7,(5,7)),(6,(6,7)),(6,(6,3))$ \\
\hline 3 & $(1,(1,2)),(1,(1,3)),(1,(1,4)),(1,(1,5)),(1,(1,6))$, \\
& $(1,(1,7)),(3,(2,3)),(4,(2,4)),(5,(2,5)),(6,(2,6))$, \\
& $(7,(2,7))$ \\
\hline 4 & $(1,(2,1)),(1,(3,1)),(1,(4,1)),(5,(3,5)),(5,(5,4))$, \\
& $(5,(5,1)),(5,(5,2)),(7,(4,7)),(7,(7,2)),(7,(7,3))$, \\
& $(6,(3,6)),(5,(5,6)),(6,(4,6)),(7,(7,6)),(7,(7,1))$ \\
\hline 5 & $(2,(2,1)),(1,(5,1)),(1,(6,1)),(1,(3,1)),(2,(2,4))$, \\
& $(2,(2,5)),(3,(3,4)),(3,(5,3)),(4,(5,4)),(3,(3,6))$, \\
& $(2,(6,2)),(7,(7,4)),(7,(7,5)),(4,(6,4)),(7,(6,7))$ \\
\hline 6 & $(2,(1,2)),(3,(1,3)),(4,(1,4)),(5,(1,5)),(2,(2,3))$, \\
& $(4,(4,3)),(2,(2,6)),(2,(2,7)),(5,(5,3)),(3,(6,3))$, \\
& $(3,(7,3)),(4,(7,4)),(5,(5,7)),(4,(4,6)),(5,(6,5))$ \\
\hline
\end{tabular}

Table 1 . An oriented incidence colouring $\vec{K}_{7}$ with six colours. 


\begin{tabular}{c|l} 
Colour Class & Vertex List \\
\hline 1 & $(6,(6,8)),(1,(2,1)),(3,(3,1)),(3,(3,2)),(2,(2,4))$, \\
& $(3,(3,4)),(4,(5,4)),(6,(2,6)),(6,(6,1)),(6,(6,4))$, \\
& $(6,(6,5)),(4,(7,4)),(8,(5,8)),(3,(5,3)),(1,(7,1))$, \\
& $(6,(7,6)),(3,(7,3)),(1,(5,1))$ \\
\hline 2 & $(8,(8,5)),(8,(8,6)),(2,(2,1)),(2,(2,3)),(8,(3,8))$, \\
& $(1,(3,1)),(4,(4,1)),(4,(3,4)),(4,(4,5)),(4,(4,7))$, \\
& $(6,(3,6)),(2,(2,6)),(4,(4,6)),(6,(5,6)),(2,(5,2))$, \\
& $(7,(5,7)),(2,(2,7))$ \\
\hline 3 & $(1,(8,1)),(2,(8,2)),(3,(8,3)),(4,(8,4)),(5,(8,5))$, \\
& $(6,(8,6)),(7,(8,7)),(1,(1,2)),(1,(1,3)),(1,(1,4))$, \\
& $(1,(1,5)),(1,(1,6)),(7,(7,2)),(7,(7,4)),(7,(7,5))$, \\
& $(7,(7,6)),(7,(7,3))$ \\
\hline 4 & $(1,(1,8)),(1,(1,7)),(2,(2,8)),(3,(3,8)),(2,(2,4))$, \\
& $(3,(4,3)),(8,(4,8)),(1,(4,1)),(3,(3,6)),(1,(6,1))$, \\
& $(2,(6,2)),(5,(7,5)),(2,(2,5)),(3,(3,5)),(5,(4,5))$, \\
& $(5,(6,5)),(2,(7,2)),(3,(3,7)),(8,(6,8)),(8,(7,8))$ \\
\hline 5 & $(8,(1,8)),(4,(4,8)),(5,(5,8)),(7,(7,8)),(8,(2,8))$, \\
& $(3,(1,3)),(4,(1,4)),(3,(2,3)),(4,(4,2)),(4,(4,3))$, \\
& $(5,(2,5)),(5,(5,1)),(3,(6,3)),(4,(6,4)),(5,(5,3))$, \\
& $(7,(2,7)),(7,(7,1)),(5,(5,6)),(7,(6,7))$ \\
\hline 6 & $(8,(8,1)),(8,(8,2)),(8,(8,3)),(8,(8,4)),(8,(8,7))$, \\
& $(2,(1,2)),(2,(3,2)),(5,(1,5)),(6,(1,6)),(7,(1,7))$, \\
& $(2,(4,2)),(5,(5,2)),(6,(6,2)),(6,(6,3)),(6,(4,6))$, \\
& $(5,(3,5)),(7,(4,7)),(5,(5,4)),(5,(5,7)),(6,(6,7))$, \\
& $(7,(3,7))$ \\
\hline &
\end{tabular}

Table 2. An oriented incidence colouring $\vec{K}_{8}$ with six colours. 

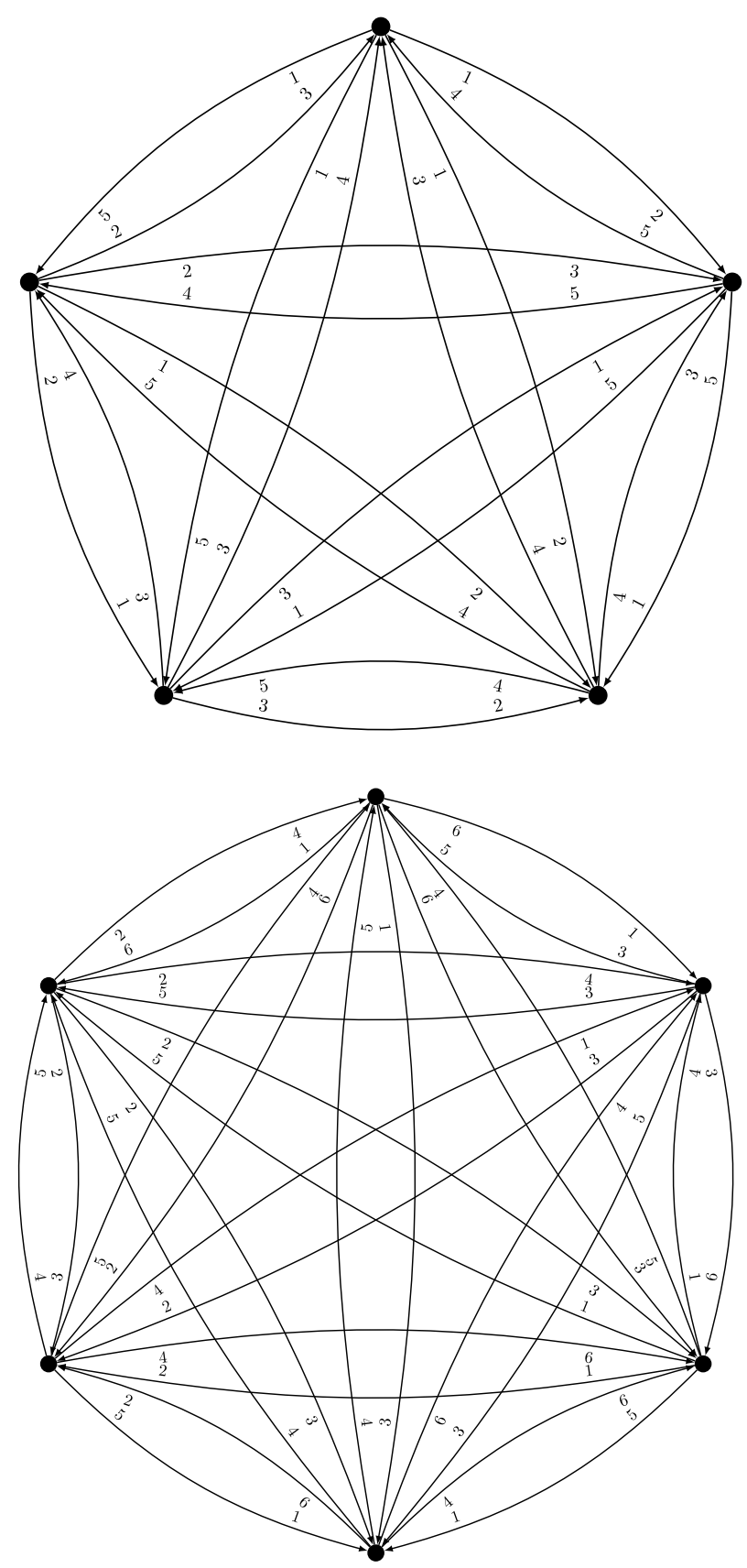

Figure 1. Oriented incidence colourings of $\vec{K}_{4}, \vec{K}_{5}, \vec{K}_{6}$ with the minimum number of colours. The colouring of $\vec{K}_{4}$ is obtained by deleting any vertex in the colouring of $\vec{K}_{5}$. 